\title{
Fast design of arbitrary length loops in proteins using InteractiveRosetta
}

\author{
William F. Hooper ${ }^{1,2}$, Benjamin D. Walcott ${ }^{2}$, Xing Wang ${ }^{3}$ and Christopher Bystroff $2,4^{*}$ (D)
}

\begin{abstract}
Background: With increasing interest in ab initio protein design, there is a desire to be able to fully explore the design space of insertions and deletions. Nature inserts and deletes residues to optimize energy and function, but allowing variable length indels in the context of an interactive protein design session presents challenges with regard to speed and accuracy.

Results: Here we present a new module (INDEL) for InteractiveRosetta which allows the user to specify a range of lengths for a desired indel, and which returns a set of low energy backbones in a matter of seconds. To make the loop search fast, loop anchor points are geometrically hashed using $C \alpha-C \alpha$ and $C \beta-C \beta$ distances, and the hash is mapped to start and end points in a pre-compiled random access file of non-redundant, protein backbone coordinates. Loops with superposable anchors are filtered for collisions and returned to InteractiveRosetta as poly-alanine for display and selective incorporation into the design template. Sidechains can then be added using RosettaDesign tools.
\end{abstract}

Conclusions: INDEL was able to find viable loops in 100\% of 500 attempts for all lengths from 3 to 20 residues. INDEL has been applied to the task of designing a domain-swapping loop for T7-endonuclease I, changing its specificity from Holliday junctions to paranemic crossover (PX) DNA.

Keywords: Indel, Bystroff, InteractiveRosetta, Rosetta, PyRosetta, T7 endonuclease I, Protein design, Simulation, Loop modeling

\section{Background}

Computational protein design is the task of finding an energy-optimal amino acid sequence for a backbone structure. Simplifying assumptions, such as fixed backbone atoms and discrete side chain conformations $[1,2]$, have been necessary because of the prohibitive size of the computational sequence search space. But, as computational resources improve, simplifying assumptions are falling away in favor of increased accuracy [3]. No longer is the backbone assumed to be fixed [4], and side chain conformations are no longer assumed to fall into discrete distributions [5]. The design process is increasingly looking like the natural process of random mutation and energetic selection. But we still assume that the template does not undergo deletions or insertions. To make protein

\footnotetext{
*Correspondence: bystrc@rpi.edu

${ }^{2}$ Department of Biology, Rensselaer Polytechnic Institute, Troy, NY, USA

${ }^{4}$ Department of Computer Science, Rensselaer Polytechnic Institute, Troy, NY, USA

Full list of author information is available at the end of the article
}

design even more like molecular evolution, we should allow the algorithm to explore the space of insertions and deletions (indels).

Searching the space of indels presents a host of computational problems. The expanded search space now includes the locations of the 'anchor' residues, defined as the last residue before and the first residue after the indel. Additionally, the length is variable, as is the sequence. Out of the necessity for computationally efficiency, we propose a hierarchy of searches. When indels occur naturally, they create a mutational "hotspot" around the gap position. This results in a viable but energetically suboptimal species immediately after indel introduction, increasing the probability of energetically advantageous mutations. If we want our algorithm to follow this natural process, our first step should be to explore the space of loop lengths without considering the side chains. This is the problem we address in this paper. The related problems of searching backbone flexibility and side chain mutation space are already solved by existing algorithms for energy

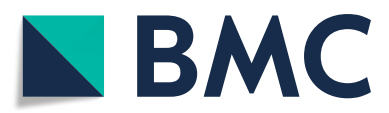

(c) The Author(s). 2018 Open Access This article is distributed under the terms of the Creative Commons Attribution 4.0 International License (http://creativecommons.org/licenses/by/4.0/), which permits unrestricted use, distribution, and reproduction in any medium, provided you give appropriate credit to the original author(s) and the source, provide a link to the Creative Commons license, and indicate if changes were made. The Creative Commons Public Domain Dedication waiver (http://creativecommons.org/publicdomain/zero/1.0/) applies to the data made available in this article, unless otherwise stated. 
minimization and protein design $[6,7]$, respectively. If we are justified in separating the indel search from the sequence design, then we may be able to open up a new world of protein design in which the chain length is now a variable.

Current approaches to loop modeling are either physicsbased or template based. The physics-based algorithms include kinematic closure (KIC), fragment assembly and analytic loop closure (FALC), molecular dynamics (MODELLER), and many more [8-11]. KIC was inspired by a technique in robotics for positioning joints with constraints. Random loop subfragments are selected to define 6 pivot points, then values for the 6 pivots are solved such that the loop is closed. KIC is usually used in the context of a Monte Carlo algorithm with simulated annealing $[8,9]$. FALC is a hierarchical approach that employs KIC. Database fragments are found for 5 and 7 amino acid residue segments. These are inserted using KIC, then scored and ranked using a force field. Rotamers are added and the fragments are again scored and ranked [10]. In contrast, MODELLER [11] randomizes the loop atomic positions, then uses all-atom energy minimization and molecular dynamics to predict the conformation, but this method is CPU intensive. Other notable methods include GalaxyLoop [12, 13], RAPPER [14, 15], and PLOP/HLP [16-18].

INDEL is a template-based loop design algorithm that draws loops from a list of high-resolution crystal structures precompiled into a random-access database. Loops are indexed by anchors using $C \alpha-C \alpha$ and $C \beta-$ $\mathrm{C} \beta$ distances, and the two-dimensional distance bins are sorted and mapped to a second-level index which can be calculated directly from the anchor point $C \alpha-C \alpha$ and $C \beta-C \beta$ distances. This two-level look-up approach allows for fast retrieval without distance calculations and without searching the database. Candidate loops are pruned in a second pass if backbone collisions are found, and in a third pass the remaining candidate loops are energy minimized and scored using Rosetta. The final candidates may be used as templates for design using fixbb or other Rosetta-Design protocols.

As proof of concept, we have applied INDEL to a comparative modeling case in which a two-residue insertion was made in the core of green fluorescent protein, and the structure was subsequently solved by X-ray crystallography (AT-GFP, PDB 4LW5) [19]. The algorithm quickly identified a database loop that closely matched the experimentally determined one. We also show that INDEL can be applied to a system that contains multiple chains, protein and DNA together, and a system which contains homo-dimeric symmetry, where two copies of the loop are designed simultaneously.

\section{Methods}

\section{Database structure}

The loop database structure is inspired by the constanttime speed and key-value access of a hash table. Here, three keys are used to access loops: the distance between loop anchor $C \beta$ 's $(\AA)$, anchor $C \alpha$ 's $(\AA)$, and loop length (residues). Matching each of these two distances assures that the anchor residues of a loop are both the right distance apart and are in the right relative orientation.

A goal of many hash table implementations is to avoid "collisions", where multiple keys map to the same location in the table. However, in this case, collisions are simply many database loops that map to the same anchor positions; here we want to retrieve them all. Allowable distances range from 0 to $50 \AA$, with a resolution of $0.1 \AA$. The fine-grained binning of loops allows the program to dynamically control the number of loops returned.

The first step in constructing the loop database was to build a repository of protein structures. Coordinates were drawn from the Top 8000 dataset, a curated set of 8000 high-quality crystal structures whose purpose was to update the MolProbity software [20].

Each residue was reduced to a 70-byte binary record containing PDB ID, chain, residue type, residue number, and coordinates for the atoms $\mathrm{N}, \mathrm{C} \alpha, \mathrm{C}, \mathrm{O}$, and $\mathrm{C} \beta$. Residues were renumbered sequentially to avoid complications due to insertion numbering. When a glycine was encountered, a $C \beta$ position was calculated using Kabsch's algorithm [21]. All residues from all proteins were concatenated into a single, random access file (file " $\mathrm{C}$ ", pdblist.dat, $128.5 \mathrm{MB})$.

An additional two random access files were constructed to perform the look-up. The first (file "A", grid.dat , $40 \mathrm{MB}$ ) is a three dimensional array, 500x500x20 in size, where the axes correspond to $C \alpha C \alpha$ distance, $C \beta C \beta$ distance, and the anchor separation distance. Each entry in the array is a tuple: a pointer to a record in file $\mathrm{B}$, and the total number of contiguous records starting from that one. The second database file (file "B", looplist.dat, 271.4 MB) consists of tuples: a pointer to the beginning of a loop in file $\mathrm{C}$, and the loop's length in residues. These files are akin to a library's card catalogue where each drawer of the catalogue represents a pair of $C \alpha C \alpha$ and $C \beta C \beta$ distances. Inside each drawer of this catalogue are twenty cards indicating where loops of a desired length can be found for those distances. A similar hashing scheme exists in Rosetta's LoopHash protocol, where the PDB was broken into fragments and hashed according to a 6-dimensional rigid body transform required to superimpose one anchor residue on the other [22].

File B was created from file $\mathrm{C}$ by iterating over record numbers for all intra-chain anchor pairs with separation distances from 3 to 19 , and sorting them by $\mathrm{C} \alpha \mathrm{C} \alpha$ distance, $C \beta C \beta$ distance, and separation. File A was created 
from file $B$ by reading and counting the number of records in bins of width $0.1 \AA$ in $C \alpha C \alpha$ distance and $C \beta C \beta$ distance and bins of width 1 in sequence separation. Finally, file A was populated at each grid point with the file B record number for the start of a list of contiguous file $C$ records, along with the length of that list.

\section{Database lookups and loop insertion}

A full walk-through of the INDEL loop design process is provided in Supplementary Data. To begin an INDEL database lookup from within InteractiveRosetta [23], the user first sets constraints for the search. Specifically, anchor residues are chosen, a range of allowable loop lengths, the minimum and maximum number of results to return. INDEL pull loops from the database and superposes the anchor coordinates. Loops are immediately rejected if they do not superimpose better than an RMSD cutoff, if they collide with the target structure backbone atoms, or if they are structurally redundant with respect to earlier results in the search. INDEL writes out the search results, which are subsequently inserted via PyRosetta's AnchoredGraftMover module. Each completed model is ranked by the Rosetta scoring function, and the top candidates are returned to the user for viewing. Upon selection of a loop, the side chains may be designed using the Protein Design (Rosetta's Fixbb) protocol, and the energy may be minimized using the Energy Minimization protocol.

\section{Results \\ Timing}

Fast retrieval of viable loop coordinates is essential for an interactive modeling program, and the program must run reasonably fast on a standard laptop with as few as one CPU. Hashed retrieval of loops is a fast, constanttime lookup, since no search is taking place. Most of the delay comes from the need to calculate distances between loop and target atoms, which follows a low-order polynomial $\left(O\left(n^{2}\right)\right)$. Further delay may depend on the location of the loop, since a more crowded environment would entail testing more loops to find one with no collisions. But in benchmarking the code using a variety of loop length, we found the loops were returned in under $13 \mathrm{~s}$ in the vast majority of cases, and never did it take over $70 \mathrm{~s}$ to return an answer, regardless of length or location (Fig. 1).

\section{Native length loop reconstruction}

INDEL is capable of inserting loops of lengths between 2 and 20 residues long. For each of these 19 lengths, a random loop region of the same length was selected for INDEL design from a random protein within the VAST nr-PDB database [24, 25]. The RMSD of the inserted loop to the original loop was then assessed. The loop was rejected if the shortest distance between a backbone atom of the inserted loop and a backbone atom of the target protein was below INDEL's collision cutoff $(4.0 \AA$ by default). All loops, whether accepted or rejected, were sorted by the backbone atom RMSD to the native loop and the ROC curve was calculated [26, 27] to assess the ability of the algorithm to preferentially keep low-RMSD loops. The $p$-value is the probability of getting the ROC value or better after scrambling the data. Accepted loops were sorted by RMSD and the distributions are summarized in Fig. 2. Lowest-RMSD examples are often within $1 \AA$ RMSD (Fig. 3).

\section{Modeling an engineered insertion.}

INDEL was used to model a loop that was engineered into GFP, converting a loop containing a cis peptide bond to a 2-residue longer loop that has all trans peptide bonds. The variant, called All-trans-GFP or AT-GFP, was subsequently solved by X-ray crystallography (PDBid 4LW5) [19]. The algorithm quickly identified a database loop that closely matched the experimentally determined one. Figure 4 shows the original structure, the $\mathrm{X}$-ray structure of the variant, and the loop predicted by INDEL.

\section{Designing a linker for a domain-swapped dimer.}

INDEL has been used in this lab to design linkers between globular domains. The enzyme T7 endonuclease I (T7 endoI) cuts DNA at Holliday junctions (HJ) [28, 29], but our desire is to design a version of the enzyme that cuts paranemic crossover (PX) DNA [30]. The latter is a DNA tetraplex that has unique distances and orientations between fissile phosphate backbone positions. If $\mathrm{T} 7$ endoI could be engineered to have the correct spacing and orientation between its two binding sites, then the enzyme specificity could be optimized to recognize PX instead of HJ. Figure 5 shows the results of loop design. In this case, two-fold symmetry was generated for each result of the loop search tom complete domain-swapped homo-dimer structure. Two-fold symmetry was enforced during the subsequent collision checking but not during energy minimization (energy minimization is not part of the INDEL protocol).

\section{Discussion}

InteractiveROSETTA has previously been described in [23]. As a protocol within InteractiveROSETTA, INDEL may be invoked from the protocol menu on the left panel. From here the user selects all the parameters for the INDEL run, such as anchor residues and loop length. The resulting loops are then output in energy score order for the user to review. Each loop may be viewed before selecting one to design (Fig. 6).

INDEL can consistently find a loop with a low RMSD to the native loop when the loop length is constrained to the native, as long as the loop length is 12 or less (Fig. 2). For longer loops, the current database is not 


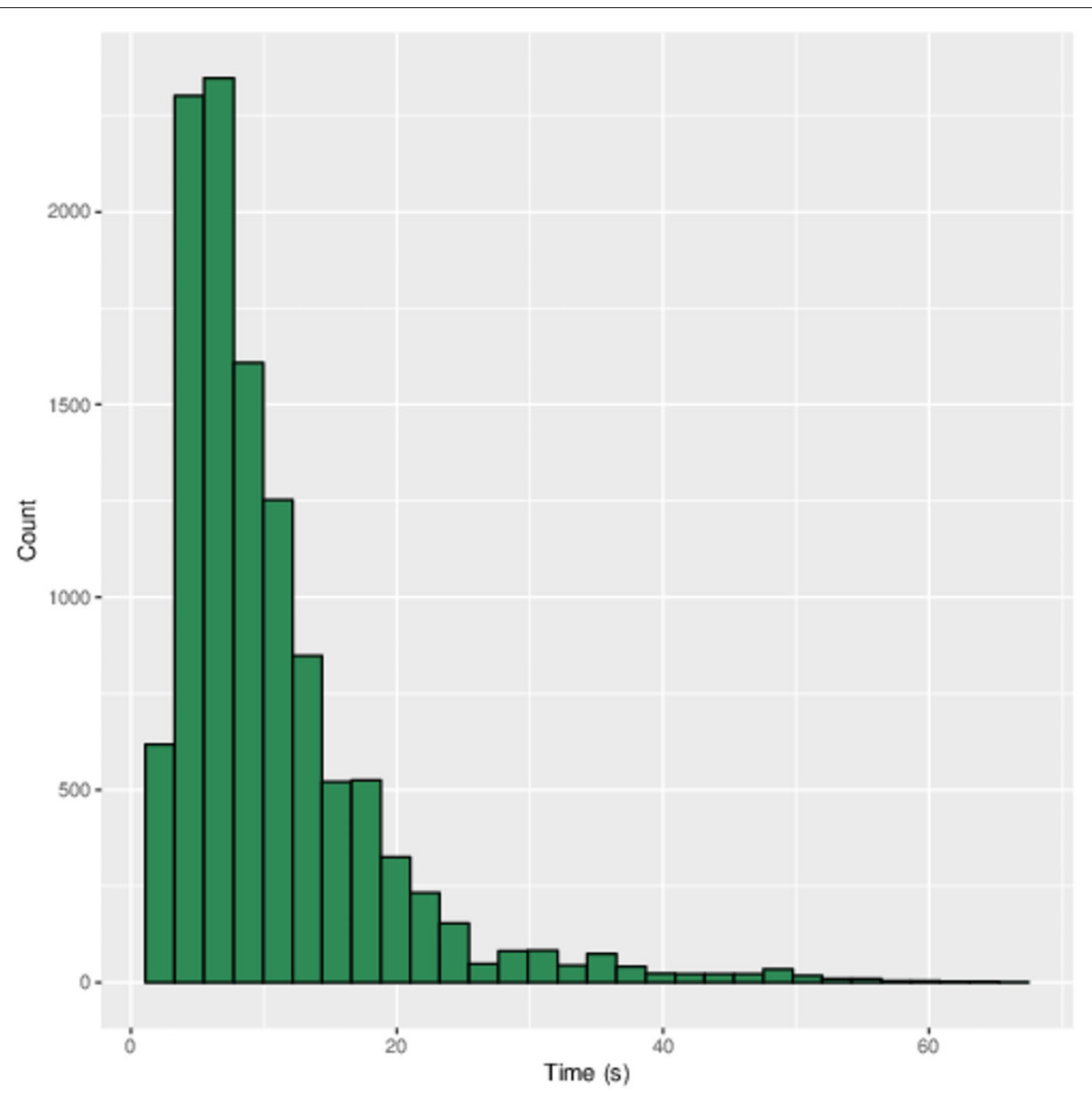

Fig. 1 INDEL insertion times. This histogram depicts the amount of time spent on each loop insertion for scaffolds of varying size via INDEL. The vast majority (75th percentile) of loop insertions occur within $13 \mathrm{~s}$

sufficiently complete to reliably return a low RMSD loop. Additionally, as the length of the loop inserted increases, so does the median RMSD of an inserted loop. The decreasing success rate with length is to be expected as the degrees of freedom of a loop increase with its sequence length. It is not likely that expanding the loop database by adding more known protein structures would help, since to improve the loop search the new proteins would have to contain new and different loop structures and novel loops appear increasingly rarely as the PDB expands. It might be possible to improve performance by allowing flexibility in the loop at the point of collision detection, but this would slow the response time.

In previously published experiments, Loophash [22], KIC [8], and Rosetta's fragment-based loop builder [31] were used to insert a 12-residue loop in a 202residue protein. Loophash takes $2 \mathrm{~s}$, Rosetta $23 \mathrm{~s}$, and KIC $260 \mathrm{~s}$ on average to perform these operations [22]. INDEL takes $10.6 \mathrm{~s}$ on average to insert a single loop. The slower constant-time search for INDEL versus Loophash is expected because INDEL searches the additional dimension of loop length.

\section{Conclusions}

The new method provides fast/best solutions for loops of different lengths, and from there on an expert user makes the choice about which is the best loop and sequence to use. The user selection can then be refined with RosettaDesign or other tools (see Additional file 1: Figures S1-S9).

Our success in designing a fast lookup for variable length loops sets up the next challenge in variable-length protein design, that of energetic identification of the best loop and sequence. InteractiveRosetta already includes modules for protein design using fixed backbone (bbfix) and flexible backbone (KIC, backrub) approaches. As such, the approach to loop selection would be to apply a flexible backbone protein design script for each of the candidate loops, and select based on energy. The performance of energetic selection would be benchmarked 


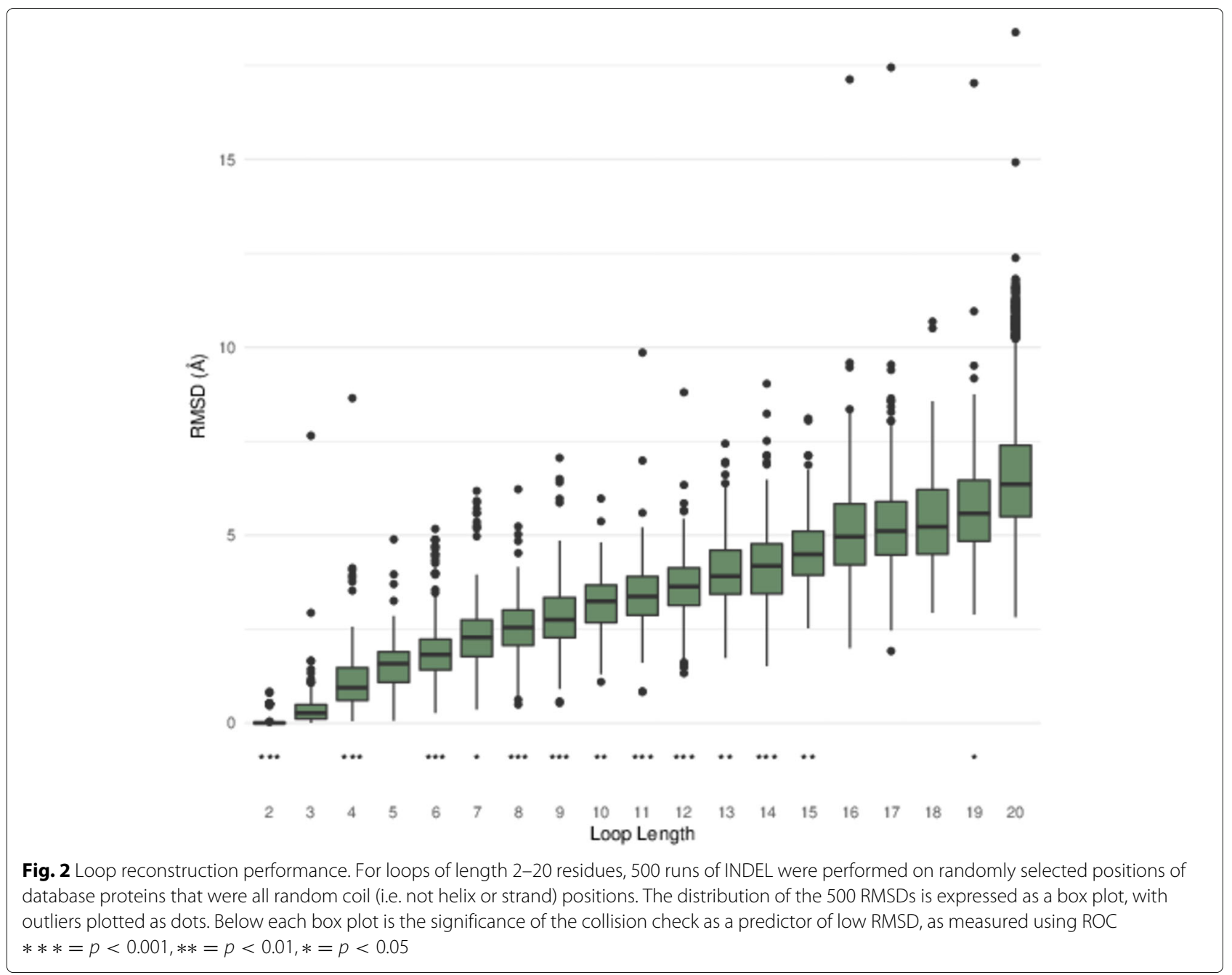

using known engineered loops or natural indels of known structure.

In the T7 endol linker-loop remodeling, expanding the search space to variable lengths was essential for success. The anchor residues of the loop corresponded to docked monomers on the phosphodiester backbone of PX DNA, instead of T7 endol's native substrate, the Holliday junction. Modeling experiments suggest that T7 endoI's native-length linker peptide would be highly strained when T7 endoI is forced to bind PX DNA (unpublished). INDEL identified linker loops for T7 endoI that can better accommodate the PX DNA phosphodiester backbone conformation and potentially improve its specificity for PX DNA over Holliday junctions (Fig. 5).

This new tool enables the exploration of the space of insertions and deletions in the context of interactive protein design. The process could also be automated as a means to explore the ways a protein could evolve in length.
To do this, we would need to establish a pipeline for energy minimization and protein design, but this is easily done in Rosetta (see Additional file 1). The resulting model could then cycle back through INDEL many times, producing an artificial evolutionary pathway.

\section{Availability and requirements}

- Project name: InteractiveRosetta / INDEL

- Project home page: https://github.com/schenc3/ InteractiveROSETTA/releases https://github.com/ schenc3/InteractiveROSETTA/releases

- Operating system(s): Windows, macOS, Ubuntu Linux

- Programming language: Python/C++

- Other requirements: PyRosetta 3 (http://www. pyrosetta.org/dowhttp://www.pyrosetta.org/dow)

- License: GNU GPL v2.0

- Any restrictions to use by non-academics: PyRosetta license required for PyRosetta dependency 

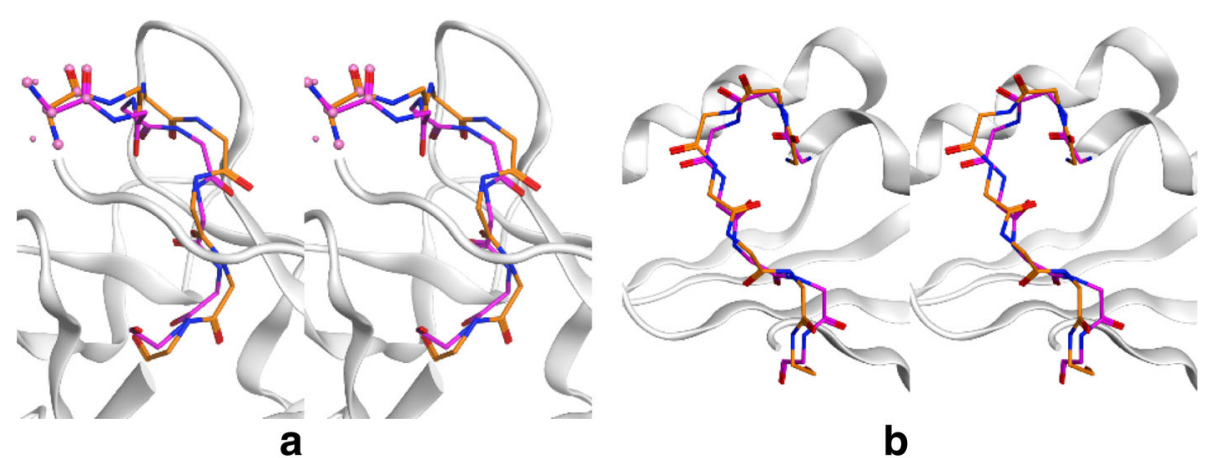

b
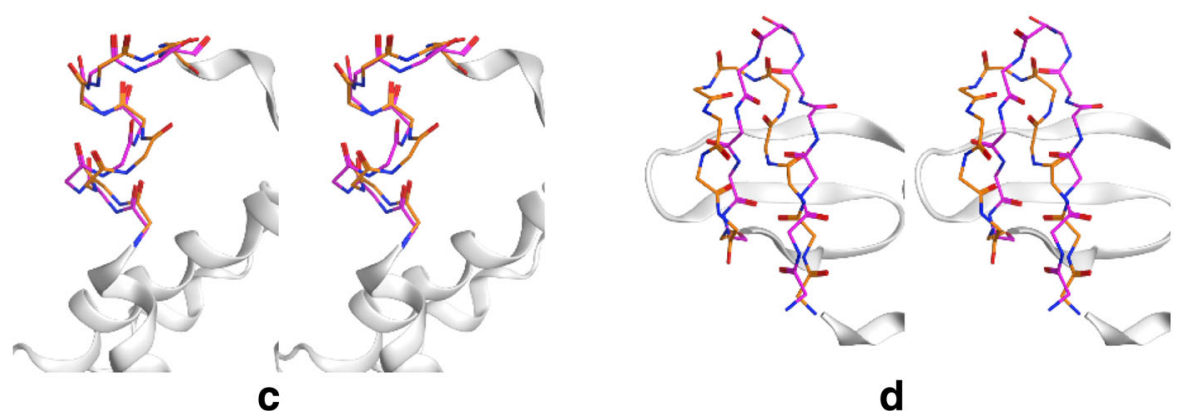

Fig. 3 Example loop reconstructions. Stereo images show loops of native length compared to the native loop for lengths a 5 (1jlx $91-95$ ), b 7 (1 eay 214-220), c 9 (3gqb 465-473), and d 11 (1s72 37-47). Native loops are in orange. Designed loops in purple

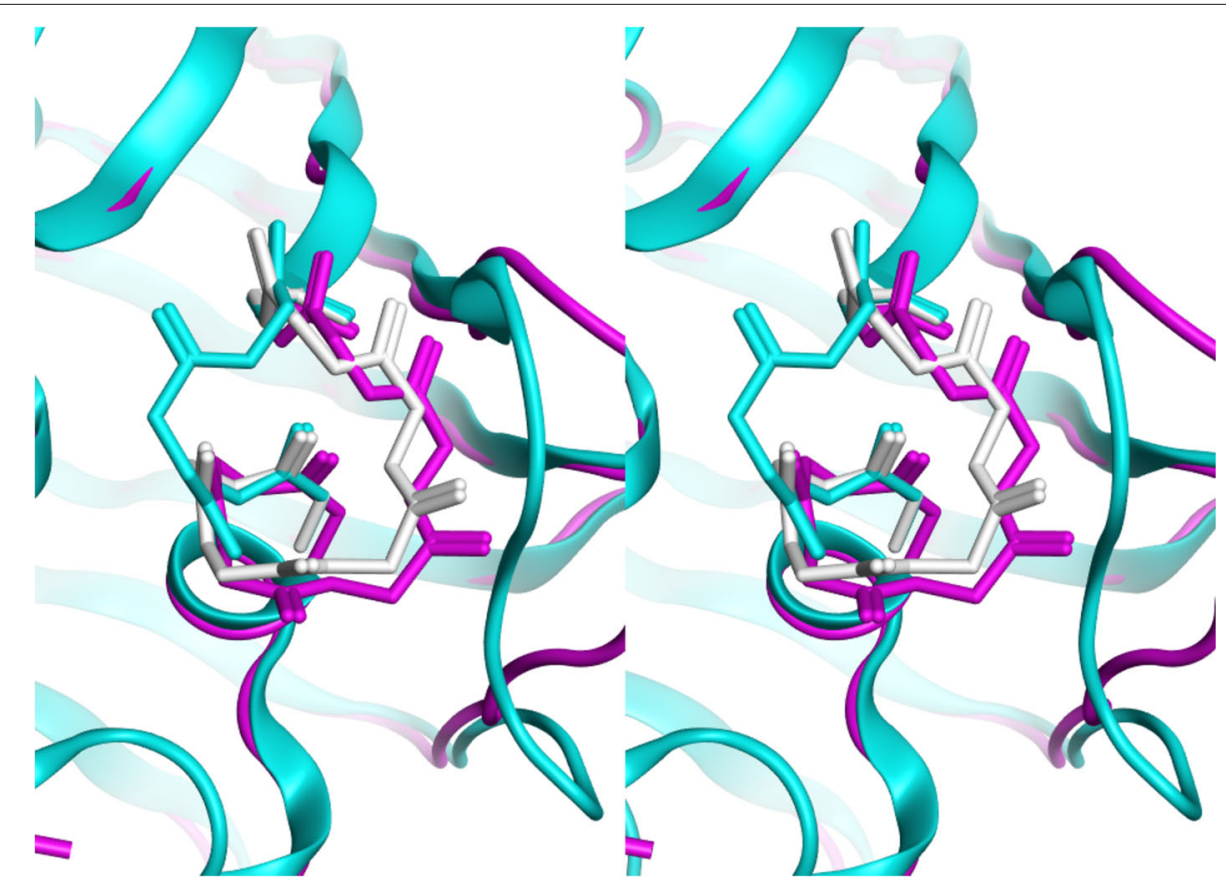

Fig. 4 AT loop reconstruction. Stereo image showing superfolder GFP near the 88-MP-89 cis-peptide bond (cyan ribbon, bonds). Into the wild-type template a 6-residue loop was inserted using INDEL. The lowest RMSD resulting loop (white) closely matches the experimentally determined structure of "All-trans" GFP (magenta) 

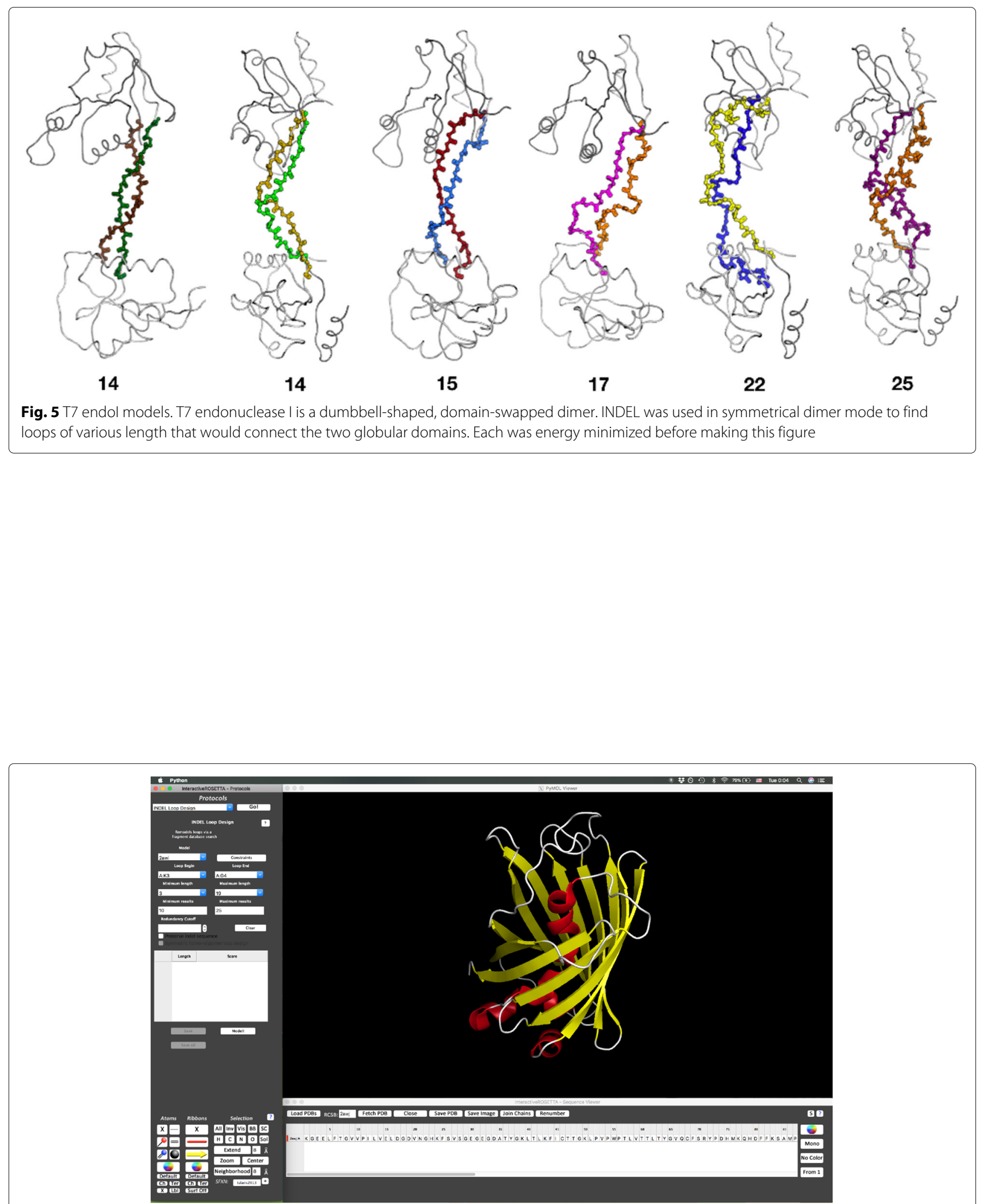

Fig. 6 INDEL design window. An example of INDEL operation within InteractiveROSETTA using PDB 2AWJ. In the left window, the user selects the protein, anchor residues, a range of loop lengths, and the number of results desired. Additionally, the user can opt to retain the source sequence of loops used and/or enforce symmetry. Results are then listed in the table in the left window and can be viewed or saved before selecting one for further design 


\section{Additional file}

Additional file 1: Supplementary Figures for "Fast Design of Arbitrary Length Loops in Proteins Using InteractiveRosetta". Storyboard walk-through of loop design using INDEL. (DOCX 3798 kb)

\section{Abbreviations}

AT GFP: All-trans green fluorescent protein; HJ: Holliday junction; PX DNA: Paranemic crossover deoxyribonucleic acids; RMSD: Root mean squared deviation; T7 endol: T7 endonuclease I

\section{Acknowledgements}

The authors acknowledge Donna E. Crone for critical comments, and the Rosetta community for the foundational software on which INDEL was built.

\section{Funding}

This work was supported by NIH grant 1R01GM099827 to CB. The funding body played no role in the design of the study, interpretation of data or writing of the manuscript.

\section{Availability of data and materials}

The datasets used and/or analyzed during the current study are available from the corresponding author on reasonable request.

\section{Authors' contributions}

WFH wrote the InteractiveRosetta module and the scripts to generate the loop database and wrote the paper. BDW debugged the module and database and performed benchmarking experiments and $\mathrm{PX} / \mathrm{T} 7$ endol docking simulations and wrote parts of the paper. XW performed experiments determining the binding interface of T7 endol with PX DNA. CB conceived the project, directed code development, generated Figs. 3, 4 and 5, and wrote part of the paper. All authors read and approved the final version of the manuscript.

\section{Ethics approval and consent to participate}

Not applicable.

\section{Consent for publication}

Not applicable.

\section{Competing interests}

The authors declare that they have no competing interests.

\section{Publisher's Note}

Springer Nature remains neutral with regard to jurisdictional claims in published maps and institutional affiliations.

\section{Author details}

${ }^{1}$ Emmes Corporation, Rockville, Washington, MD, USA. ${ }^{2}$ Department of Biology, Rensselaer Polytechnic Institute, Troy, NY, USA. ${ }^{3}$ Department of Chemistry and Chemical Biology, Rensselaer Polytechnic Institute, Troy, NY, USA. ${ }^{4}$ Department of Computer Science, Rensselaer Polytechnic Institute, Troy, NY, USA.

Received: 17 May 2018 Accepted: 29 August 2018

Published online: 24 September 2018

\section{References}

1. Desmet J, Maeyer MD, Hazes B, Lasters I. The dead-end elimination theorem and its use in protein side-chain positioning. Nature. 1992;356(6369):539-42. https://doi.org/10.1038/356539a0.

2. Dunbrack RL, Karplus M. Backbone-dependent Rotamer Library for Proteins Application to Side-chain Prediction. J Mol Biol. 1993;230(2): 543-74. https://doi.org/10.1006/jmbi.1993.1170.

3. Gainza P, Nisonoff HM, Donald BR. Algorithms for protein design. Curr Opin Struct Biol. 2016;39:16-26. https://doi.org/10.1016/j.sbi.2016.03.006.

4. Davis IW, Arendall WB, Richardson DC, Richardson JS. The Backrub Motion: How Protein Backbone Shrugs When a Sidechain Dances. Structure. 2006;14(2):265-74. https://doi.org/10.1016/j.str.2005.10.007.

5. Gainza P, Roberts KE, Donald BR. Protein Design Using Continuous Rotamers. PLoS Comput Biol. 2012;8(1):1002335. https://doi.org/10.1371/ journal.pcbi.1002335.
6. Liu Y, Kuhlman B. RosettaDesign server for protein design. Nucleic Acids Res. 2006;34(Web Server):235-8. https://doi.org/10.1093/nar/gkl163.

7. Gainza P, Roberts KE, Georgiev I, Lilien RH, Keedy DA, Chen C-y, Reza F Anderson AC, Richardson DC, Richardson JS, Donald BR. Methods in Enzymology. In: Keating AE, editor. San Diego: Elsevier; 2013. p. 87-107. Chap. 5. https://doi.org/10.1016/B978-0-12-394292-0.00005-9. http://linkinghub.elsevier.com/retrieve/pii/B9780123942920000059.

8. Mandell DJ, Coutsias EA, Kortemme T. Sub-angstrom accuracy in protein loop reconstruction by robotics-inspired conformational sampling. Nat Methods. 2009;6(8):551-2. https://doi.org/10.1038/nmeth0809-551.

9. Stein A, Kortemme T. Improvements to Robotics-Inspired Conformational Sampling in Rosetta. PLoS ONE. 2013;8(5):e63090. https:// doi.org/10.1371/journal.pone.0063090

10. Ko J, Lee D, Park H, Coutsias EA, Lee J, Seok C. The FALC-Loop web server for protein loop modeling. Nucleic Acids Res. 2011;39(SUPPL. 2): 210-4. https://doi.org/10.1093/nar/gkr352.

11. Fiser $A$, Do RKG, Sali A. Modeling of loops in protein structures. Protein Sci. 2000;9(9):1753-73. https://doi.org/10.1110/ps.9.9.1753.

12. Ko J, Park H, Heo L, Seok C. GalaxyWEB server for protein structure prediction and refinement. Nucleic Acids Res. 2012;40(W1):294-7. https:// doi.org/10.1093/nar/gks493.

13. Shin $\mathrm{W}-\mathrm{H}$, Lee $\mathrm{GR}$, Heo L, Lee $H$, Seok C. Prediction of Protein Structure and Interaction by GALAXY Protein Modeling Programs. Bio Des. 2014;2:01-11.

14. DePristo MA, de Bakker PIW, Lovell SC, Blundell TL. Ab initio construction of polypeptide fragments: Efficient generation of accurate, representative ensembles. Proteins Struct Funct Genet. 2003;51(1):41-55. https://doi. org/10.1002/prot.10285.

15. De Bakker PIW, DePristo MA, Burke DF, Blundell TL. Ab initio construction of polypeptide fragments: Accuracy of loop decoy discrimination by an all-atom statistical potential and the AMBER force field with the generalized born solvation model. Proteins Struct Funct Genet. 2003;51(1):21-40. https://doi.org/10.1002/prot.10235.

16. Jacobson MP, Pincus DL, Rapp CS, Day TJF, Honig B, Shaw DE, Friesner RA. A hierarchical approach to all-atom protein loop prediction. Proteins Struct Funct Bioinforma. 2004;55(2):351-67. https://doi.org/10. 1002/prot.10613.

17. Zhu K, Pincus DL, Zhao S, Friesner RA. Long loop prediction using the protein local optimization program. Proteins Struct Funct Bioinforma. 2006;65(2):438-52. https://doi.org/10.1002/prot.21040.

18. Sellers BD, Zhu K, Zhao S, Friesner RA, Jacobson MP. Toward better refinement of comparative models: Predicting loops in inexact environments. Proteins Struct Funct Bioinforma. 2008;72(3):959-71. https://doi.org/10.1002/prot.21990.

19. Rosenman DJ, Huang Y-m, Xia K, Fraser K, Jones VE, Lamberson CM, Van Roey P, Colón W, Bystroff C. Green-lighting green fluorescent protein: Faster and more efficient folding by eliminating a cis-trans peptide isomerization event. Protein Sci. 2014;23(4):400-10. https://doi. org/10.1002/pro.2421.

20. Chen VB, Arendall WB, Headd JJ, Keedy DA, Immormino RM, Kapral GJ, Murray LW, Richardson JS, Richardson DC. MolProbity: All-atom structure validation for macromolecular crystallography. Acta Crystallogr D Biol Crystallogr. 2010;66(1):12-21. https://doi.org/10.1107/ S0907444909042073.

21. Kabsch W. A solution for the best rotation to relate two sets of vectors Acta Crystallogr A. 1976;32(5):922-3. https://doi.org/10.1107/ S0567739476001873.

22. Tyka MD, Jung $K$, Baker D. Efficient sampling of protein conformational space using fast loop building and batch minimization on highly parallel computers. J Comput Chem. 2012;33(31):2483-91. https://doi.org/10. 1002/jcc.23069

23. Schenkelberg CD, Bystroff C. InteractiveROSETTA: A graphical user interface for the PyRosetta protein modeling suite. Bioinformatics. 2015;31(24):4023-5. https://doi.org/10.1093/bioinformatics/btv492.

24. Gibrat JF, Madej T, Bryant SH. Surprising similarities in structure comparison. Curr Opin Struct Biol. 1996;6(3):377-85. https://doi.org/10. 1016/S0959-440X(96)80058-3.

25. Madej T, Lanczycki CJ, Zhang D, Thiessen PA, Geer RC, Marchler-Bauer A Bryant SH. MMDB and VAST+: Tracking structural similarities between macromolecular complexes. Nucleic Acids Res. 2014;42(D1):297-303. https://doi.org/10.1093/nar/gkt1208. 
26. Robin X, Turck N, Hainard A, Tiberti N, Lisacek F, Sanchez JC, Müller M. pROC: An open-source package for R and S+ to analyze and compare ROC curves. BMC Bioinformatics. 2011;12. https://doi.org/10.1186/14712105-12-77.

27. Hanley JA, MCNeil BJ. The meaning and use of the area under a receiver operating characteristic (ROC) curve. Radiology. 1982;143(1):29-36. https://doi.org/10.1148/radiology.143.1.7063747.

28. Hadden JM, Convery Ma, Déclais A-CC, Lilley DMJ, Phillips SEV. Crystal structure of the Holliday junction resolving enzyme T7 endonuclease I. Nat Struct Biol. 2001;8(1):62-7. https://doi.org/10.1038/83067.

29. Hadden JM, Déclais A-C, Carr SB, Lilley DMJ, Phillips SEV. The structural basis of Holliday junction resolution by T7 endonuclease I, Nature. 2007;449(7162):621-4. https://doi.org/10.1038/nature06158.

30. Shen Z, Yan H, Wang T, Seeman NC. Paranemic crossover DNA: a generalized Holliday structure with applications in nanotechnology. J Am Chem Soc. 2004;126(6):1666-74.

31. Qian B, Raman S, Das R, Bradley P, McCoy AJ, Read RJ, Baker D. Highresolution structure prediction and the crystallographic phase problem. Nature. 2007;450(7167):259-64. https://doi.org/10.1038/nature06249.

Ready to submit your research? Choose BMC and benefit from:

- fast, convenient online submission

- thorough peer review by experienced researchers in your field

- rapid publication on acceptance

- support for research data, including large and complex data types

- gold Open Access which fosters wider collaboration and increased citations

- maximum visibility for your research: over $100 \mathrm{M}$ website views per year

At BMC, research is always in progress.

Learn more biomedcentral.com/submissions 